Ambiente \& Água - An Interdisciplinary Journal of Applied Science
ISSN 1980-993X - doi:10.4136/1980-993X
www.ambi-agua.net
E-mail: ambi.agua@gmail.com

\title{
Análise de procedimentos para seleção de áreas prioritárias em programas de pagamento por serviços ambientais hídricos
}

\author{
doi:10.4136/ambi-agua.1782
}

Received: 19 Oct. 2015; Accepted: 15 Dec. 2015

\section{Ana Feital Gjorup ${ }^{1 *}$; Elaine Cristina Cardoso Fidalgo ${ }^{2}$; Rachel Bardy Prado ${ }^{2}$; Azeneth Eufrasino Schuler ${ }^{2}$}

\author{
${ }^{1}$ Universidade Federal Fluminense (UFF), Niterói, RJ, Brasil \\ Programa de Pós-Graduação em Engenharia de Biossistemas (PGEB) \\ ${ }^{2}$ Empresa Brasileira de Pesquisas Agropecuárias (Embrapa solos), Rio de Janeiro, RJ, Brasil \\ Departamento de Núcleo de Geomática (Ngeo) \\ *Autor correspondente: e-mail: ana_feital@hotmail.com, \\ elaine.fidalgo@embrapa.br, rachel.prado@embrapa.br, \\ marisolschuler@gmail.com
}

\section{RESUMO}

A abordagem de serviços ecossistêmicos tem se mostrado promissora para a avaliação das interações entre ecossistema e sociedade ao integrar conceitos ambientais e socioeconômicos o que requer conhecimento interdisciplinar. Porém, sua aplicação encontra limitações devido a lacunas de informação que orientem a tomada de decisão. Diante disso o presente trabalho foi desenvolvido buscando contribuir para a aplicação de princípios de serviços ecossistêmicos, no processo decisório em gestão de recursos hídricos. Seu objetivo é identificar procedimentos e metodologias empregados para a tomada de decisão visando a seleção de áreas prioritárias a serem contempladas em projetos ou programas de compensação por serviços ambientais. Para tal buscou-se na literatura técnica e científica publicações que descrevessem métodos e experiências para seleção de áreas prioritárias; identificaram-se as etapas-chave empregadas no processo de seleção de áreas prioritárias; em seguida fez-se um levantamento dos procedimentos adotados para cada etapa-chave, nos textos reunidos; e, por fim, as informações coletadas foram analisadas e classificadas. Considerando o universo amostral do presente trabalho, observou-se que a seleção de áreas prioritárias foi realizada com base no emprego direto dos critérios previamente determinados. A aplicação de indicadores, bem como a análise espacial, ainda são práticas pouco empregadas. Deve-se ressaltar, contudo, que a maioria dos documentos analisados não se propunha a descrever o processo de seleção de áreas prioritárias em detalhe, o que pode ter resultado em algumas omissões. Embora essas condições possam trazer limitações para as análises efetuadas neste trabalho, os resultados apresentados permitem identificar os principais objetivos, ações e critérios empregados para a seleção de áreas prioritárias em programas/projetos de compensação por serviços ambientais.

Palavras-chave: compensação por serviços ambientais, serviços ecossistêmicos, serviços ambientais hídricos, suporte à decisão. 


\title{
Analysis of selection procedures to determine priority areas for payment for water ecosystem services programs
}

\begin{abstract}
The approach of ecosystem services has shown promise for the evaluation of interactions between ecosystems and society, integrating environmental and socioeconomic concepts which require interdisciplinary knowledge. However, its usefulness in decision making is limited due to information gaps. This study was therefore developed in order to contribute to the application of principles of ecosystem services in the decision-making for water resources management. It aims to identify procedures and methodologies used for decision-making in order to select priority areas to be included in projects or compensation programs for environmental services. To do so, we searched technical and scientific literature describing methods and experiences used to select priority areas. Key steps in the process of selecting priority areas were identified; then a survey was conducted of the procedures adopted for each key step considering the literature selected; and, finally, the information collected was analyzed and classified. Considering the study's sample, we noted that the selection of priority areas was based on the direct use of predetermined criteria. The use of indicators and spatial analyses are practices still scarcely employed. We must highlight, however, that most of the analyzed documents did not aim to describe the process of selecting priority areas in detail, which may have resulted in some omissions. Although these conditions may limit the analysis in this study, the results presented here allow us to identify the main objectives, actions and criteria used to select priority areas for programs or compensation projects for environmental services.
\end{abstract}

Keywords: compensation for ecosystem services, ecosystem services, decision support, water ecosystem services.

\section{INTRODUÇÃO}

Os ecossistemas proporcionam uma variedade de produtos e serviços que contribuem, direta ou indiretamente, com o bem-estar humano (FAO, 2007). Apesar de sua importância para a vida, paradoxalmente, muitos estão seriamente ameaçados pelos impactos causados pelas diversas atividades humanas. Segundo Millennium Ecosystem Assesment (MEA, 2003) os ecossistemas e recursos naturais têm sido substancialmente explorados, degradados e destruídos no último século como consequência do crescimento econômico global.

Diante dos desafios colocados pelos êxitos e fracassos advindos do crescimento econômico, da necessidade de conciliar questões relativas ao desenvolvimento econômico e questões relativas ao meio ambiente, diversas iniciativas internacionais foram tomadas a partir da década de 1970 (Comissão Mundial Sobre o Meio Ambiente e Desenvolvimento, 1991; FAO, 2007; MEA, 2003). Em junho de 2001, o programa Millennium Ecosystem Assessment (MEA), lançado pelo então Secretário-Geral das Nações Unidas, Kofi Annan, analisou como as mudanças nos serviços dos ecossistemas têm afetado o bem-estar humano e podem afetar as pessoas nas próximas décadas e que tipos de respostas podem ser adotadas em escala local, nacional ou global para melhorar a gestão dos ecossistemas e contribuir, assim, para o bemestar humano e a redução da pobreza (MEA, 2003).

Peralta (2014) destaca que para construir uma nova mentalidade que vise à sustentabilidade ambiental, deverá ser reestruturado o processo de incentivos econômicos que conduzem os seres humanos a degradar o meio ambiente, de forma que as pessoas sejam orientadas a tomar decisões e desenvolver estilos de vida sustentáveis. Os instrumentos econômicos são um importante mecanismo para orientar, de maneira sustentável, as atividades dos agentes econômicos e distribuir de uma maneira mais adequada os custos 
socioambientais.

Neste contexto, visando reduzir a degradação dos ecossistemas, o conceito de serviços ecossistêmicos (SE) se tornou tema central no planejamento conservacionista (Koschke et al., 2012; Fisher e Turner, 2008). A abordagem de SE segundo Burkhard et al. (2009) tem sido bastante empregada, dentre vários motivos, por seu caráter integrador, que possibilita pesquisas interdisciplinares, reunindo conceitos ambientais e socioeconômicos.

Observa-se na literatura o emprego de dois diferentes termos: serviços ecossistêmicos e serviços ambientais. Os serviços ecossistêmicos, segundo definição de MEA (2003), refletem os benefícios diretos e indiretos providos pelo funcionamento dos ecossistemas, sem a interferência humana. Já nos serviços ambientais, os benefícios gerados estão associados também a ações de manejo do homem nos sistemas naturais ou agroecossistemas. Englobam tanto os serviços proporcionados ao ser humano por ecossistemas naturais (os serviços ecossistêmicos), quanto os providos por ecossistemas manejados ativamente pelo homem (agricultura agroflorestal, manejo florestal, reflorestamento) (Guedes e Seehusen, 2011). Muitas vezes os autores não diferenciam os termos, sendo comum o emprego de serviços ecossistêmicos na literatura internacional e serviços ambientais na nacional. Neste trabalho, empregamos o termo serviços ambientais a todos os serviços proporcionados pelos ecossistemas, manejados ou não pelo homem.

Considerando que o homem pode influenciar positivamente a oferta de serviços ambientais a partir da sua escolha em adotar práticas agrícolas diversificadas e sustentáveis (Muradian et al., 2009), tem-se intensificado a criação e aplicação de mecanismos de incentivo a essas práticas. Diversos autores salientam as vantagens de aplicação de mecanismos econômicos de gestão ambiental que utilizam sistemas de incentivos e desincentivos, financeiros ou fiscais visando orientar o mercado de forma que as diversas atividades econômicas sejam realizadas de maneira sustentável (Peralta, 2014; Rincón-Ruiz, 2014). Peralta (2014) ressalta o uso crescente do sistema de Pagamento por Serviços Ambientais (PSA) como um instrumento econômico de gestão ambiental com o intuito de internalizar externalidades ambientais positivas. No Brasil, segundo Pagiola et al. (2013), nos últimos anos tem sido observado um número crescente de esquemas de PSA. Um dos exemplos de emprego de mecanismo de PSA voltado à melhoria e proteção de recursos hídricos é o Programa Produtor de Água, da Agência Nacional de Águas, do Ministério do Meio Ambiente do Brasil. O Programa se efetiva por meio de articulações e parcerias entre instituições das esferas municipal, estadual, federal e privada, visando o desenvolvimento da política de PSA como um instrumento de incentivo aos produtores rurais que adotarem, de forma voluntária, práticas voltadas à conservação de água e solo (ANA, 2012).

Uma questão que se coloca a programas de PSA bem como a todos os programas e projetos que atuam através de mecanismos de incentivo é onde atuar prioritariamente. Essa questão é colocada em termos econômicos uma vez que os recursos financeiros para aplicação nesses programas são sempre limitados, mas também envolve a priorização para direcionar esforços de ação para áreas onde as intervenções produzirão os melhores benefícios (Orsi et al., 2011).

As vantagens da aplicação do conceito de serviços ecossistêmicos ou ambientais têm sido bastante exploradas. Do ponto de vista analítico, como salienta De Groot (2006), pode prover uma relação sistemática dos componentes e processos ecossistêmicos mais importantes e a dependência que as sociedades humanas têm deles. Entretanto, Burkhard et al. (2009) destacam que a aplicação do conceito de bens e serviços ecossistêmicos em nível de paisagem encontra limitações devido às lacunas de dados apropriados e de sua quantificação, ou seja, falta informação diretamente aplicável em escala regional ou local que oriente a tomada de decisão.

Nas últimas duas décadas, diferentes categorias de SE foram propostas buscando uma 
melhor compreensão dos mesmos (MEA, 2003; Haines-Young e Potschin, 2010), embora não haja consenso em torno de um único modelo de classificação. Haines-Young e Potcshin (2010) afirmam que dificilmente uma lista simples e genérica de classificação dos SE reuniria toda a diversidade de ecossistemas e regiões. Entretanto a classificação dos SE tem sido empregada como guia, orientando levantamentos e monitoramentos desses serviços. No caso dos serviços relacionados aos recursos hídricos, descrevem-se dois tipos: a) os serviços de provisão de água, que asseguram o abastecimento de água para a população; e os de regulação hídrica, que se relacionam às características regulatórias dos processos ecossistêmicos que refletem na quantidade e qualidade da água (MEA, 2003).

O presente trabalho foi desenvolvido buscando contribuir para a aplicação de princípios de serviços ecossistêmicos no processo decisório em gestão de recursos hídricos.

Seu objetivo geral foi identificar, compilar e analisar procedimentos e metodologias empregados para a tomada de decisão, visando à seleção de áreas prioritárias a serem contempladas em projetos ou programas de compensação por serviços ambientais.

\section{MATERIAL E MÉTODOS}

O trabalho foi desenvolvido como uma pesquisa exploratória descritiva, baseando-se no levantamento, análise e síntese de métodos propostos e de experiências relatadas destinadas à seleção de áreas prioritárias para pagamento ou outras formas de compensação pelo fornecimento de serviços ambientais, com maior enfoque nos serviços ambientais relacionados à provisão e regulação hídrica.

O trabalho iniciou com a identificação de palavras-chave para selecionar publicações ou relatórios que descrevessem métodos e experiências para a seleção de áreas prioritárias.

As palavras-chave foram empregadas em buscas em uma base de publicações sobre serviços ambientais hídricos organizada e descrita por Lyra et al. (2014). Essa base reunia à época 278 documentos sobre serviços ambientais. As principais fontes de informação são publicações catalogadas, documentos governamentais, relatórios de organizações internacionais e projetos locais em páginas da internet, incluindo artigos científicos, relatórios, manuais e comunicados que expressam experiências relevantes no tema. Dentre os documentos, há referências a programas de compensação por serviços ambientais no Brasil e em outras partes do mundo, sendo priorizadas as relacionadas aos serviços ambientais hídricos. Á época desta pesquisa, a base de publicações reunia documentos datados de 1996 a 2014, porém os documentos selecionados por sua relação com o tema abordado são do período entre 2006 e 2014.

A organização da informação relativa a procedimentos e métodos para a seleção de áreas prioritárias foi feita segundo as etapas deste processo, sendo dividida em:

- definição dos objetivos do processo de seleção de áreas prioritárias;

- definição das ações propostas para intervenção;

- estabelecimento de critérios para a seleção;

- realização de ponderação aplicada aos critérios;

- aplicação de método para integração de dados; e

- aplicação de método de validação dos resultados.

$\mathrm{Na}$ análise dos documentos foram compiladas as informações sobre cada um dos itens acima, quando existentes. No caso de experiências que se referiam a programas ou projetos de compensação por serviços ambientais, também foram registrados o nome do programa, o país, e quando informada, a localidade.

Para a organização e a análise das informações, foram adotados como referências o trabalho desenvolvido por Bennett et al. (2012) e o manual do Programa Produtor de Água da 
Agência Nacional de Águas (ANA, 2012). O trabalho de Bennett et al. (2012) foi adotado por trazer informações atualizadas oriundas da análise de vários programas de PSA em bacias hidrográficas que ocorrem em todo o mundo. O documento reúne informações de 205 programas ativos, sendo 76 em desenvolvimento. O manual do Programa Produtor de Água da Agência Nacional de Águas (ANA, 2012) também foi escolhido como referência porque estabelece orientações para o programa nacional que tem como objetivo geral apoiar projetos de PSA de proteção hídrica que visem promover a melhoria da qualidade e a ampliação da oferta das águas e a regularização da vazão dos corpos hídricos.

\section{RESULTADOS E DISCUSSÃO}

\subsection{Levantamento inicial}

A aplicação das palavras-chave para levantamento nos 278 documentos na base de dados permitiu selecionar previamente 57 deles que poderiam fazer referência à seleção de áreas prioritárias para programas de compensação por serviços ambientais. A análise dos 57 documentos resultou na identificação de 17 que apresentavam procedimentos para a seleção de áreas prioritárias. Um desses documentos, fornecido pela TNC no formato de relato de experiência, não foi publicado.

Os 17 documentos analisados (ANA, 2012; Bernardes, 2010; Bochner, 2010; Instituto Estadual de Florestas, 2011; Haaren et al., 2012; Micol et al., 2008; Guatemala, 2012; Mota et al., 2013; Muñoz-Piñaa et al., 2008; Naidoo e Ricketts, 2006; Padovezi et al., 2011; Zhang e Pagiola, 2011; Peralta, 2014; Pagiola et al., 2013; Pereira, 2013; Rio de Janeiro et al., 2012; Silva et al., 2013; Ulber et al., 2011), descreveram 29 métodos de seleção de áreas prioritárias, provenientes de seis países. No Brasil foram identificadas 20 experiências de programas que envolveram a seleção de áreas prioritárias.

Importante destacar que a base de dados consultada tem como principal foco experiências relacionadas aos serviços ambientais hídricos. Dos 29 métodos levantados, 21 tinham como foco recursos hídricos; sendo que seis incluíam biodiversidade e dois incluíam carbono. Esses oito relatos de experiências que não tratavam de forma explícita os recursos hídricos foram incluídos por contribuírem com a descrição de procedimentos para seleção de áreas prioritárias.

\subsection{Objetivos para seleção de áreas prioritárias}

Foram classificados três tipos de objetivos: 1) objetivos diretamente relacionados à melhoria dos serviços hídricos, 2) objetivos visando proporcionar benefícios socioeconômicos e 3) objetivos visando proporcionar melhoria de outros serviços ambientais (Figura 1). Cabe salientar que alguns documentos analisados não descreviam claramente seus objetivos. Eles foram recuperados ao longo da descrição do projeto ou programa, com base nas ações propostas, no processo para a seleção de áreas de intervenção ou mesmo nos resultados obtidos.

Os objetivos diretamente relacionados à melhoria dos serviços hídricos foram os mais relatados, o que era esperado, uma vez que este trabalho priorizou a análise de experiências da aplicação de serviços ambientais em gestão de recursos hídricos.

Considerando esse conjunto de objetivos, os mais citados são aqueles relacionados diretamente à provisão de água de qualidade: regular o fluxo hídrico e melhorar a qualidade da água. Os demais objetivos - controlar sedimentos, melhorar a infiltração da água no solo, conservar as bacias hidrográficas, proteger as nascentes e controlar a poluição da água embora expressem forte relação com os serviços e sejam capazes de alterá-los, são menos citados. 

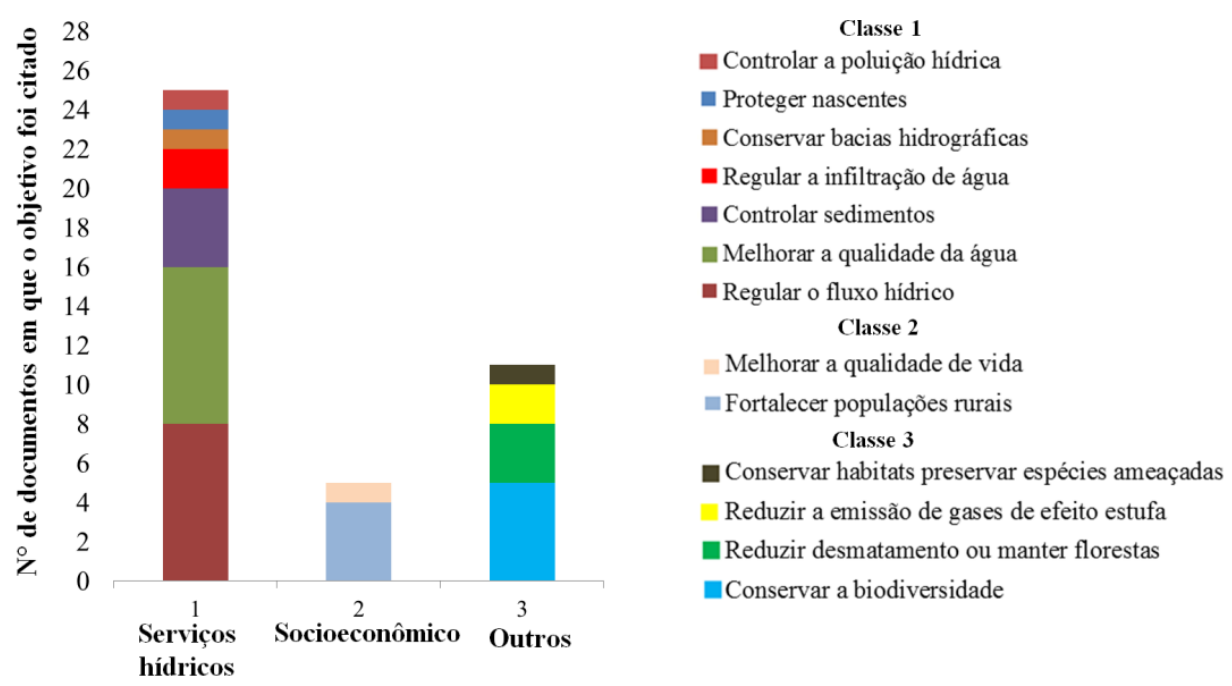

Figura 1. Objetivos do processo de seleção de áreas prioritárias observados nos documentos analisados.

Considerando os aspectos socioeconômicos, predominou o objetivo "fortalecer populações rurais", que reflete as características dos programas descritos na bibliografia considerada, os quais envolvem prioritariamente as populações rurais como fornecedoras de serviços ambientais. Como exemplo deste tipo de objetivo podemos citar "promover a permanência das populações rurais em suas comunidades”.

Dentre os objetivos que visam proporcionar melhoria de outros serviços ambientais, os mais frequentes foram conservar a biodiversidade (citado em cinco documentos), reduzir desmatamento ou manter florestas (3) e reduzir a emissão de gases de efeito estufa (GEE) (2).

\subsection{Ações propostas para intervenção}

As ações de intervenção foram analisadas segundo classificação proposta por Bennett (2012). A Figura 2 mostra o número de ações de intervenção propostas nos documentos analisados segundo essa classificação e a Tabela 1 apresenta um detalhamento dessas ações.

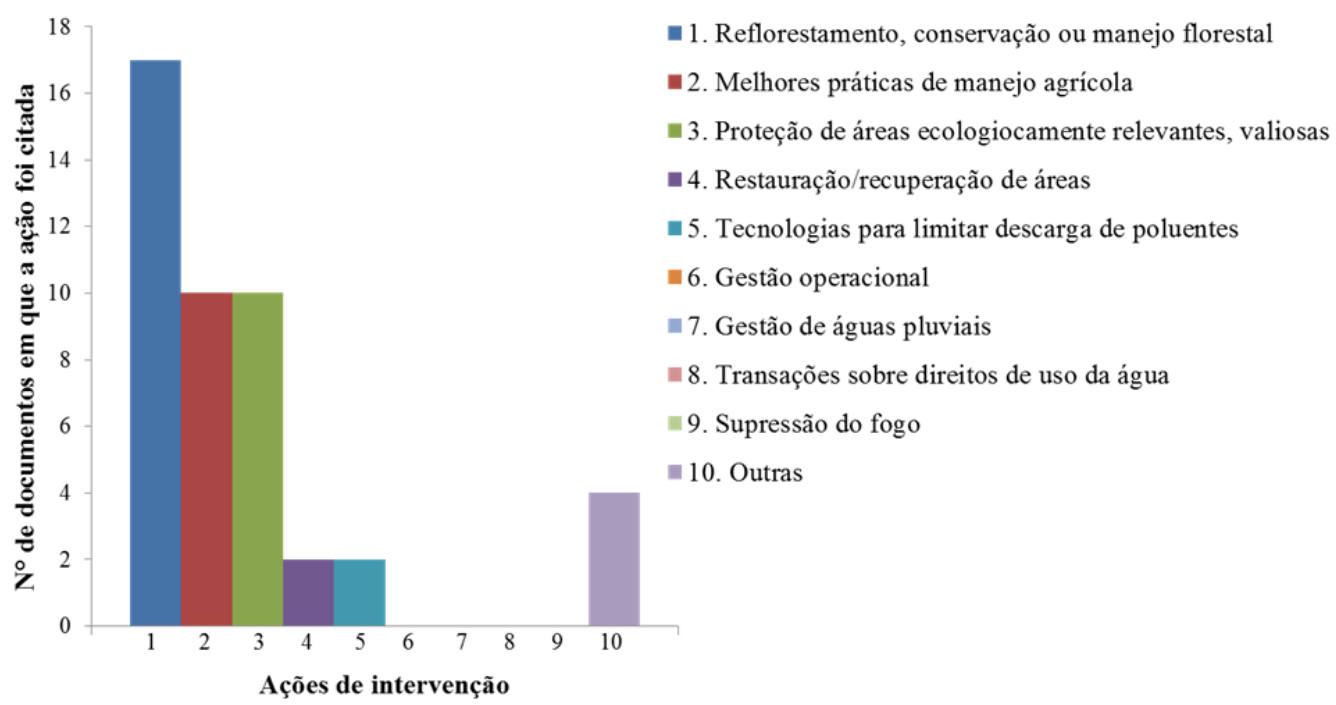

Figura 2. Ações propostas para intervenção observadas nos documentos analisados. 
Tabela 1. Ações propostas para intervenção.

\section{Ações}

Número de

casos

\section{Reflorestamento, conservação ou manejo florestal}

Manutenção ou proteção de áreas de floresta ou vegetação natural

Reflorestamento ou condução de regeneração com espécies nativas

Envolvem os dois tipos de ação (manutenção/proteção ou implantação de cobertura vegetal)

\section{Desenvolvimento de melhores práticas agrícolas}

Desenvolvimento de melhores práticas agrícolas

Apoiar/incentivar a produção em bases sustentáveis (aumento da diversidade

de plantas cultivadas, sistemas agroflorestais, manejo florestal)

\section{Proteção de áreas ecologicamente relevantes}

Manutenção/proteção ou implantação de cobertura vegetal em Área de

Preservação Permanente (APP)

Manutenção/proteção ou implantação de cobertura vegetal em APP e/ou

Reserva Legal (RL)

Manutenção/proteção ou implantação de cobertura vegetal em outras áreas (proximidade com unidade de conservação)

\section{Recuperação ou restauração de áreas}

Recuperação ou restauração de áreas degradadas

5. Tecnologias para limitar descarga de poluentes

Controle de disposição de resíduos sólidos e tratamento de efluentes

\section{Outras}

Medidas para conservação da biodiversidade

Medidas para redução das disparidades de desenvolvimento social

Não foram observadas nos documentos analisados as ações de intervenção classificadas como gestão operacional, gestão de águas pluviais, transações sobre direitos de uso da água e supressão do fogo.

\subsection{Critérios para priorização}

Os critérios para a seleção de áreas prioritárias ao desenvolvimento das ações propostas levantados nos documentos analisados foram divididos em dois grupos: os de elegibilidade e os de priorização de áreas à intervenção.

Os critérios de elegibilidade são aplicados para selecionar o público-alvo conforme as demandas do programa. A elegibilidade, em geral, é uma análise prévia, não estabelece prioridades, mas sim condições prévias para a participação, sendo empregada de forma independente ao processo de seleção de áreas prioritárias, por exemplo, a disposição do produtor em participar, ou a obrigatoriedade do produtor residir na bacia selecionada.

O levantamento dos critérios de priorização de áreas à intervenção revelou a diversidade destes. Alguns foram estabelecidos para identificar regiões prioritárias, que podem ser bacias hidrográficas, municípios ou outras delimitações definidas a priori pelo programa.

Outros critérios foram definidos para selecionar propriedades ou posses cujas áreas apresentam condições prioritárias para as ações propostas. Apresentamos a seguir os critérios 
levantados, destacando os casos em que são aplicados especificamente para a seleção de propriedades ou posses.

\subsubsection{Critérios relacionados aos recursos hídricos:}

- Importância da bacia para abastecimento;

- Ocorrência de alta escassez de água ou alto risco de inundação;

- Existência de conflito pelo uso da água;

- $\quad$ Nível de degradação da bacia e presença de usos conflitantes com conservação;

- $\quad$ Índice de urbanização;

- Potencial de redução de aporte de sedimentos mediante intervenção;

- $\quad$ Suscetibilidade a erosão;

- Características físicas, como limiar de declividade, limiar de gradiente altitudinal, limiar de densidade de drenagem;

- Limiar de média pluviomética; e

- Considerando a seleção de propriedades ou posses: localização na bacia hidrográfica (montante ou jusante), presença e/ou estado de conservação de APP, localização em áreas cársticas, localização em áreas de recarga, presença de cobertura vegetal, proximidade com Unidade de Conservação (UC), limiar de declividade.

\subsubsection{Critérios relacionados à biodiversidade:}

- Presença de alta biodiversidade;

- Localização em relação a áreas protegidas e corredores biológicos;

- Ocorrência de espécies ameaçadas ou em risco de extinção;

- Tamanho mínimo de fragmentos, tamanho e distância de fragmentos;

- Potencial de conectividade;

- Valor da área de vegetação natural - estabelecido segundo seu potencial de ocorrência de carne de caça, de madeira comercializável, potencial para bioprospecção, valor de existência, armazenamento de carbono, custo considerando conversão para agricultura; e

- Considerando a seleção de propriedades ou posses: proximidade com unidades de conservação; presença de áreas de vegetação natural ou proporção de área de vegetação natural; presença de vegetação em estágios mais avançados de sucessão; presença de reserva legal averbada; presença de vegetação em percentual superior ao mínimo destinado à reserva legal; e diversidade de plantas cultivadas.

\subsubsection{Critérios relacionados ao carbono:}

- Condição da área em 1990 e atualmente em relação à cobertura da vegetação nativa;

- Valor Potencial do PSA ${ }^{1}$.

\subsubsection{Critérios relacionados ao desenvolvimento social:}

- $\quad$ Potencial de redução de pobreza (índice de pobreza, concentração de pobreza na região, índice de desenvolvimento social); e

- Considerando a seleção de propriedades ou posses: produtores familiares; proprietários que recebam compensações por outro programa executado dentro da

${ }^{1}$ O Valor Potencial do PSA é dado pela expressão:

Valor Potencial do PSA = Adicionalidade Potencial - Custo Potencial em que, Adicionalidade Potencial $=$ Probabilidade de desmatamento $\mathrm{x}$ Massa de Carbono $\mathrm{x}$ Valor de mercado de carbono e Custo Potencial $=$ Custo de oportunidade de conservação. 
área; propriedades ou posses objeto de aplicação de financiamento na linha de conservação ambiental ou agroecológica; propriedades/posses vinculadas a projetos públicos de inclusão social no campo; propriedades/posses vinculadas a políticas públicas destinadas à juventude rural; propriedades/posses que participem de projetos associativos de produção; propriedades ou posses pertencentes a povos tradicionais; e propriedades ou posses localizadas em UCs.

\subsubsection{Outros critérios:}

- Áreas declaradas como prioritárias em regulamentação específica (legislação, planos diretores de bacia ou municipais) ou definidas em levantamentos previamente realizados;

- Existência de instituições parceiras com atuação local;

- Existência de projetos ambientais desenvolvidos por entidades locais;

- Município com equipe atuante na área de meio ambiente; e

- Considerando a seleção de propriedades ou posses: coerência do produtor com escopo do projeto ou cumprimento da legislação ambiental; e existência de práticas amigáveis de uso da terra - conservação do solo, produção sem uso de agrotóxico, prática de controle biológico, produção agroecológica.

O Programa Produtor de Água (ANA, 2012) descreve critérios para a seleção de subbacias para o desenvolvimento das ações do programa. Segundo o programa, a sub-bacia é elegível se atender a pelo menos um dos critérios descritos a seguir, sendo prioritária aquela que atender ao maior número deles:

- ser manancial de abastecimento de água para uso urbano ou industrial;

- $\quad$ ser manancial de fornecimento de água para a geração de energia elétrica;

- estar inserida em bacias hidrográficas que já tenham os instrumentos de gestão, previstos na Lei 9.443/97, implementados;

- estar inserida em uma bacia hidrográfica cujo Plano de Recursos Hídricos identifique problemas de poluição difusa de origem rural, erosão e déficit de cobertura vegetal em áreas legalmente protegidas;

- $\quad$ ter um número mínimo de produtores rurais interessados que possa viabilizar a aplicação do Programa;

- estar em situação de conflito de uso dos recursos hídricos; e

- estar sujeita a eventos hidrológicos críticos recorrentes.

Nas sub-bacias selecionadas, são realizados projetos individuais de propriedades, sendo priorizados os projetos que aportam maiores benefícios ambientais, ou seja, que alteram, de modo significativo, a qualidade da água da sub-bacia ou promovam a redução da erosão e a melhoria da infiltração de água. Em parte os critérios para a seleção de sub-bacias propostos pelo Programa Produtor de Água se assemelham aos critérios levantados nos demais documentos.

\subsection{Ponderação dos critérios}

Em relação à atribuição de peso aos critérios, observa-se que não é uma prática comum diferenciar os critérios segundo sua importância ou relevância na análise. Somente em um documento (Bochner, 2010) foi descrito o emprego de um método de ponderação. Nesse caso, foi feita uma atribuição de pesos aos critérios segundo sua importância. Os pesos, no intervalo entre 1 e 10, foram atribuídos individualmente por especialistas. Os resultados individuais foram reunidos e analisados utilizando o método Processo Analítico Hierárquico (AHP) (Saaty, 1980), para se obter o peso final para cada critério. 
A ausência de ponderação simplifica a análise, por outro lado, ela a restringe ao pressupor que todos os critérios têm o mesmo grau de importância. A vantagem de aplicação de ponderação é evidenciada no estudo de Bochner (2010) ao permitir considerar diferentes pesos aos critérios empregados, que podem refletir sua importância ou impacto para o alcance dos objetivos. Essa vantagem, aliada à escassez de métodos de ponderação empregados nos casos estudados, aponta para a necessidade de estudos visando a identificação e aplicação de técnicas de ponderação adequadas para a priorização de áreas de intervenção em PSA.

\subsection{Métodos de análise integrada}

O levantamento dos métodos aplicados para a análise integrada do conjunto dos critérios mostrou que há uma diversidade de abordagens. Dos 29 métodos ou procedimentos descritos, 28 descreveram algum tipo de método para integrar os critérios utilizados.

$\mathrm{Na}$ maioria dos casos, com 17 ocorrências, os critérios foram aplicados diretamente e selecionados aqueles que atendiam o maior número deles. Em quatro casos foram relatados o emprego de um indicador: a) o índice de suscetibilidade à erosão (The Nature Conservancy, 2013), b) o índice de percentual de abatimento de erosão (PAE) (Pagiola et al., 2013), c) o valor potencial da aplicação do PSA (Micol et al., 2008) e d) o aporte de sedimentos (The Nature Conservancy, 2013). Três documentos citam a sobreposição de mapas e aplicação de critérios: a) sobreposição de mapeamentos diversos (Zhang e Pagiola, 2011); sobreposição de mapeamento de suscetibilidade à erosão, principais mananciais e corredores ecológicos (relato de experiência fornecido por The Nature Conservancy em 2013); e 'área ativa de rio' que considera a manutenção da conectividade hidrológica e a variabilidade natural das áreas ripárias desde a nascente até a foz de um rio (Pagiola et al., 2013). Por último, com uma ocorrência cada, tem-se: análise custo-benefício espacial considerando estimativas dos custos de oportunidade de conservação (Naidoo e Ricketts, 2006); abordagem multicriterial em ambiente de Sistema de Informação Geográfica (SIG), empregando Combinação Linear Ponderada (CLP) (Bochner, 2010); avaliação da biodiversidade através de indicadores (Sistema MANUELA) (Haaren et al., 2012); e leilão baseado em propostas dos agricultores contendo listagem de serviços ambientais fornecidos e valor a receber (Ulber et al., 2011).

\subsection{Validação}

O emprego de métodos de validação foi observado em apenas dois casos: um que verificou a robustez aplicando outro método sobre os mesmos dados e comparando os resultados (Haaren et al., 2012); e o outro realizou testes aplicando o método em áreas nas quais havia PSA em andamento e comparou os resultados (Zhang e Pagiola, 2011).

A validação proporciona maior confiabilidade nos resultados. O número escasso de trabalhos que aplicam técnicas de validação justifica a necessidade de estudos visando a identificação, aplicação e avaliação dessas técnicas.

\section{CONCLUSÕES}

A análise dos documentos que relatam procedimentos para seleção de áreas prioritárias para programas de compensação por serviços ambientais revelou que os objetivos do processo de seleção de áreas prioritárias nem sempre são explicitados, constituindo-se em uma lacuna de informação que pode prejudicar a definição das intervenções necessárias e os critérios para seleção de locais prioritários à sua execução.

Dentre as intervenções mais frequentemente propostas para as áreas selecionadas tem-se o florestamento / reflorestamento ou manejo florestal, o desenvolvimento de melhores práticas agrícolas e a proteção de áreas ecologicamente relevantes.

A seleção de áreas prioritárias foi realizada na maior parte dos casos com base no 
emprego direto dos critérios previamente determinados. A aplicação de indicadores, bem como a análise espacial, ainda são práticas pouco empregadas.

Considerando a diversidade de critérios e a especificidade observada em alguns dos casos levantados, conclui-se que seu desenvolvimento e aplicação são bastante voltados às condições locais ou regionais ou a objetivos específicos do programa analisado.

É necessário que seja dada maior atenção à análise conjunta dos critérios, principalmente se considerarmos que pode haver alta correlação, seja positiva ou negativa, entre eles, o que pode levar a uma avaliação tendenciosa das áreas prioritárias, com sobrevalorização ou desvalorização de algumas de suas características.

Deve-se ressaltar, contudo, que a maioria dos documentos analisados não se propunha a descrever o processo de seleção de áreas prioritárias em detalhe, o que pode ter resultado em algumas omissões. Embora essas condições possam trazer limitações para as análises efetuadas neste trabalho, os resultados aqui apresentados permitem identificar os principais objetivos, ações e critérios empregados para a seleção de áreas prioritárias em programas/projetos de compensação por serviços ambientais. Além disso, indicam a necessidade urgente de que esses projetos ou programas sistematizem e relatem suas experiências na área.

\section{AGRADECIMENTOS}

Agradecemos à Embrapa pelo financiamento do projeto "Fortalecimento do conhecimento, organização da informação e elaboração de instrumentos de apoio aos Programas de Pagamentos por Serviços Ambientais Hídricos no meio rural”, no âmbito do qual este trabalho foi desenvolvido. Agradecemos a João Guimarães, da TNC, parceiro deste projeto, pelas informações fornecidas sobre o levantamento de áreas estratégicas para ações de conservação e produção de água no Espírito Santo.

\section{REFERÊNCIAS}

AGÊNCIA NACIONAL DE ÁGUAS - ANA (Brasil). Manual operativo do programa Produtor de Água. 2. ed. Brasília, 2012. 84 p.

BENNETT, G.; CARROLL, N.; HAMILTON, K. Charting new waters: state of watershed payments. Washington, DC: Forest Trends, 2012. Disponível em: <http://www.ecosystemmarketplace.com/reports/sowp2012>. Acesso em: 10 mar. 2015

BERNARDES, A. C. Pagamento por serviços ambientais: experiências brasileiras relacionadas à água. In: ENCONTRO NACIONAL DA ANPPAS, 5., 4-7 outubro 2010. Anais... Florianópolis: ANPPAS, 2010.

BOCHNER, J. K. Proposta metodológica para identificação de áreas prioritárias para recomposição florestal - estudo de caso: bacia hidrográfica do rio Macacu-RJ. 2010. 135f. Dissertação (Mestrado em Ciências) Pós-Graduação em Ciências Ambientais e Florestais, Universidade Federal Rural do Rio de Janeiro, Seropédica, 2010.

BURKHARD, B.; KROLL, F.; MÜLLER, F.; WINDHORST, W. Landscapes' capacities to provide ecosystem services: a concept for land-cover based assessments. Landscape Online, v. 15, p. 1-22, 2009.

COMISSÃO MUNDIAL SOBRE O MEIO AMBIENTE E DESENVOLVIMENTO. Nosso futuro comum. 2. ed. Rio de Janeiro: FGV, 1991. 428 p. 
DE GROOT, R. S. Function-analysis and valuation as a tool to assess land use conflicts in planning for sustainable, multi-functional landscapes. Landscape and Urban Planning, v. 75, p. 175-186, 2006. http://dx.doi.org/10.1016/j.landurbplan.2005.02.016

FOOD AND AGRICULTURE ORGANIZATION OF THE UNITED NATIONS - FAO. Land evaluation: towards a revised framework. Rome, 2007 (Land and Water Discussion Paper, n. 6).

FISHER, B.; TURNER, R. K. Ecosystem services: classification for valuation. Biological conservation, v. 141, p. 1167-1169, 2008.

http://dx.doi.org/10.1016/j.biocon.2008.02.019

GUATEMALA. Ministerio de Ambiente y Recursos Naturales. Lecciones aprendidas sobre los esquemas de compensación o pago por servicios ambientales implementados en el Corredor Seco con el apoyo del Efecto 4 del Programa Conjunto. Guatemala, 2012. 33 p.

GUEDES, F. B.; SEEHUSEN, S. E. (Orgs.). Pagamentos por serviços ambientais na Mata Atlântica: lições aprendidas e desafios. Brasília: MMA, 2011. 272 p. (Biodiversidade, n. 42)

HAAREN, C. VON; KEMPA, D.; VOGEL, K.; RÜTER S. Assessing biodiversity on the farm scale as basis for ecosystem service payments. Journal of Environmental Management, v. 113, p. 40-50, 2012. http://dx.doi.org/10.1016/j.jenvman.2012.07.033

HAINES-YOUNG, R.; POTSCHIN, M. The links between biodiversity, ecosystem services and human well-being. In: RAFAELLI, G. D.; FRID, C. L. J. (Eds.). Ecosystem ecology: a nex synthesis. Cambridge: Cambridge University Press, 2010. p. 110-139.

INSTITUTO ESTADUAL DE FLORESTAS. Diretoria de Desenvolvimento e Conservação Florestal. Bolsa verde: manual de princípios, critérios e procedimentos para a implantação da lei n.17.727 de agosto de 2008. Belo Horizonte, 2011.

KOSCHKE, L.; FÜRST, C.; FRANK, S.; MAKESCHIN, F. A multi-criteria approach for an integrated land-cover-based assessment of ecosystem services provision to support landscape planning. Ecological Indicators, v. 21, p. 54-66, 2012. http://dx.doi.org/10.1016/j.ecolind.2011.12.010

LYRA, W. D. S.; LIMA, A. P. M.; ALBUQUERQUE, R. H.; INÁCIO, M. C.; GJORUP, A. F.; MACARIO, C. G. N. M. et al. Methodological approach of a database as support to Programs of Payment for Ecosystem Water Services in Brazil. In: CONFERENCE OF THE ECOSYSTEM SERVICES PARTNERSHIP, 7., San José, CR. Anais... San José: [s.n.], 2014.

MICOL, L.; ANDRADE, J.; BÖRNER, J. Redução das emissões do desmatamento e da degradação (REDD): potencial de aplicação em Mato Grosso. Alta Floresta: Instituto Centro de Vida, 2008.

MILLENIUM ECOSYSTEM ASSESSMENT - MEA. Ecosystems and human well-being: a framework for assessment. Washington, D.C.: Island Press, 2003.

MOTA, A. M.; MENDES, A. S. R.; RIBEIRO, P. Projeto Ecocrédito - Montes Claros. In: PAGIOLA, S.; GLEHN, H. C. V.; TAFFARELLO, D. (Orgs.). Experiências de pagamento por serviços ambientais no Brasil. São Paulo: SMA/CBRN, 2013. 
MUÑOZ-PIÑAA, C.; GUEVARAB, A.; TORRESC, J. M.; BRAÑAA, J. Paying for the hydrological services of Mexico's forests: analysis, negotiations and results. Ecological Economics, v. 65, n. 4, p. 725-736, 2008. http://dx.doi.org/10.1016/j.ecolecon.2007.07.031

MURADIAN, R.; CORBERA, E.; PASCUAL, U.; KOSOY, N.; MAY, P. H. Reconciling theory and practice: an alternative conceptual framework for understanding payments for environmental services. Ecological Economics, v. 69, p. 1202-1208, 2009. http://dx.doi.org/10.1016/j.ecolecon.2009.11.006

NAIDOO, R. L.; RICKETTS, T. H. Mapping the economic costs and benefits of conservation. PLOS biology, v. 4, n. 11, p. 2153-2164, 2006. http://dx.doi.org/10.1371/journal.pbio.0040360

ORSI, F.; GENELETTI, D.; NEWTON, A. C. Towards a common set of criteria and indicators to identify forest restoration priorities: an expert panel-based approach.

Ecological Indicators, v. 11, n. 2, p. 337-347, 2011. http://dx.doi.org/10.1016/j.ecolind.2010.06.001

PADOVEZI, A.; DIEDERICHSEN, A.; VEIGA, F. (Orgs). Projeto conservador das águas passo a passo. Brasilia: The Nature Conservancy do Brasil, 2011. (Água, Clima e Floresta, v. 4).

PAGIOLA, S.; GLEHN, H. C. V.; TAFFARELLO, D. (Orgs.). Experiências de pagamento por serviços ambientais no Brasil. São Paulo: SMA/CBRN, 2013. 274 p.

PERALTA, C. E. O pagamento por serviços ambientais como instrumento para orientar a sustentabilidade ambiental: a experiência da Costa Rica. In: LAVRATTI, P.; TEJEIRO, G. (Orgs.). Direito e mudanças climáticas: pagamento por serviços ambientais: experiências locais e latino-americanas. São Paulo: Instituto O Direito por um Planeta Verde, 2014.143p. (Direito e Mudanças Climáticas, 7).

PEREIRA, P. H. Projeto conservador das águas - Extrema. In: PAGIOLA, S.; GLEHN, H. C. V.; TAFFARELLO, D. (Orgs.). Experiências de pagamento por serviços ambientais no Brasil. São Paulo: SMA/CBRN, 2013. p. 29-40.

RINCÓN-RUÍZ, A.; ECHEVERRY-DUQUE, M.; PIÑEROS, A. M.; TAPIA, C. H.; DAVID, A.; ARIAS-ARÉVALO, P. et al. Valoración integral de la biodiversidade y los servicios ecosistémicos: aspectos conceptuales y metodológicos. Bogotá, D. C.: IAvH, 2014. $151 \mathrm{p}$.

RIO DE JANEIRO. Secretaria Estadual do Ambiente; COMITÊ DE BACIA HIDROGRÁFICA DO RIO GUANDU; RIO CLARO. Prefeitura Municipal; THE NATURE CONSERVANCY; INSTITUTO TERRA DE PRESERVAÇÃO AMBIENTAL. Manual de orientação ao proponente de prestação de serviços ambientais, n' 01/12. Guandu, 2012.

SAATY, T. The analytic hierarchy process. New York: McGraw-Hill, 1980. 287p.

ULBER, L.; KLIMEK, S.; STEINMANN, H.; ISSELSTEIN, J.; GROTH, M. Implementing and evaluating the effectiveness of a payment scheme for environmental services from agricultural land. Environmental Conservation, v. 38, n. 04, p. 464-472, 2011. http://dx.doi.org/10.1017/S0376892911000385 
THE NATURE CONSERVANCY. Annual Report. 2013. Disponível em: http://www.nature.org/media/annualreport/2013-annual-report.pdf. Acesso em dez. 2015.

ZHANG, W.; PAGIOLA, S. Assessing the potential for synergies in the implementation of payments for environmental services programmes: an empirical analysis of Costa Rica. Enviromental Conservation, v. 38, n. 04, p. 406-416, 2011. http://dx.doi.org/10.1017/S0376892911000555 